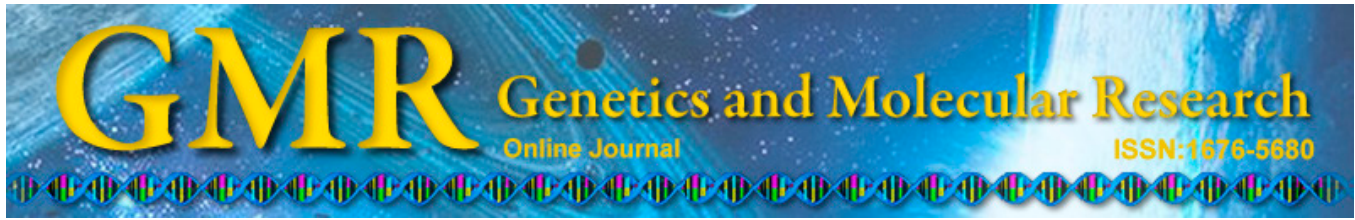

\title{
Integrated microRNA-mRNA analysis of pancreatic ductal adenocarcinoma
}

\author{
P.F. Liu ${ }^{1 *}$, W.H. Jiang ${ }^{2 *}$, Y.T. Han ${ }^{3}$, L.F. He ${ }^{4}$, H.L. Zhang ${ }^{1}$ and H. Ren ${ }^{5}$ \\ ${ }^{1}$ Key Laboratory of Cancer Prevention and Therapy, \\ Department of Lymphoma, Sino-US Center of Lymphoma and Leukemia, \\ Tianjin Medical University Cancer Institute and Hospital, \\ National Clinical Research Center for Cancer, Tianjin, China \\ ${ }^{2}$ Department of Radiotherapy, Second Hospital of Tianjin Medical University, \\ Tianjin, China \\ ${ }^{3}$ Department of Cell Biology, School of Basic Medical Sciences, \\ Tianjin Medical University, Tianjin, China \\ ${ }^{4}$ Shanxi Agricultural University, Shanxi, China \\ ${ }^{5}$ Key Laboratory of Cancer Prevention and Therapy, \\ Department of Pancreatic Cancer, \\ Tianjin Medical University Cancer Institute and Hospital, \\ National Clinical Research Center for Cancer, Tianjin, China \\ *These authors contributed equally to this study. \\ Corresponding author: H. Ren \\ E-mail: snowlpf@163.com
}

Genet. Mol. Res. 14 (3): 10288-10297 (2015)

Received December 18, 2014

Accepted May 30, 2015

Published August 28, 2015

DOI http://dx.doi.org/10.4238/2015.August.28.14

\begin{abstract}
The main aim of this study was to explore the underlying molecular mechanisms and potential target molecules of pancreatic adenocarcinoma. The miRNA (GSE32678) and mRNA (GSE32676) expression profiles of patients with pancreatic ductal adenocarcinoma and healthy controls were downloaded from the Gene Expression Omnibus database. Differentially expressed miRNA and differentially expressed genes were identified by analyzing the microarray algorithm
\end{abstract}


after data preprocessing. Functional analysis was conducted by the Database for Annotation, Visualization and Integrated Analysis. miRNA-mRNA regulation pairs were obtained in TarMir database. The node degree of $h s a-m i R-200 c$, hsa-miR-429, and $h s a-m i R-200 b$ (miRNA), and EFNB2, MYRIP, and PHF17 (mRNA) were extremely high in the miRNA-mRNA network, indicating that these miRNA and mRNA may play a key role in the development of pancreatic cancer. Our study screened out some target miRNAs and mRNAs for pancreatic ductal adenocarcinoma, which may be helpful in its diagnosis and treatment.

Key words: Pancreatic adenocarcinoma; MicroRNA; Differentially expressed gene

\section{INTRODUCTION}

Pancreatic adenocarcinoma is a highly lethal disease that is usually diagnosed at an advanced stage of disease progression. This type of cancer has very few or no effective therapies (Long et al., 2012; Siegel et al., 2014). This disease is characterized by an early local regional spread and distant metastasis. The mortality rate of pancreatic adenocarcinoma is almost $100 \%$, because of its propensity for early metastatic spreading, and its resistance to radiation and chemotherapy. Despite the vast amount of accumulated knowledge regarding tumor biology, the efficacy of the available treatment strategies for pancreatic cancer has remained largely unchanged over the past decade. The first-line agent gemcitabine has been observed to show favorable clinical responses, including reduced pain and weight gain (Matano et al., 2000); despite this, the prognosis remains dismal, with a 5-year survival rate of $1-4 \%$, and a median survival period of 4-6 months. In addition, the molecular basis for pancreatic cancer remains to be elucidated. Therefore, there is a need for further research at a molecular level to identify new molecular mechanisms or biomarkers, in an effort to improve the prognosis, diagnosis, and treatment of pancreatic cancer.

MicroRNAs (miRNA) are 18-24-nucleotide long non-coding RNA that bind to the 3 -untranslated region of target transcripts and regulate gene expression by degrading the target mRNA or inhibiting translation (Lee et al., 1993). Previous studies have indicated that miRNA serve as guidance molecules, base pairing with partial or full complementary sequences of target mRNA, leading to translational repression and/or mRNA cleavage. Research conducted to elucidate the mechanism of action of miRNA has revealed that miRNA affect stem cell differentiation, organ development, cell death, phase change in the cell cycle, signal transduction, and several diseases, including cancer (Lionetti et al., 2009; Antonini et al., 2010). Recent studies have focused on miRNA expression profiling of pancreatic adenocarcinoma. Aberrant expression of miRNA, such as miR-21 and miR-155, in the pancreas, often contributes to cancer development and invasion (Giovannetti et al., 2010; Ryu et al., 2010). On the other hand, miRNA such as miR-34 and miR-150 may also suppress the growth and malignant behavior of pancreatic cancer cells (Liu et al., 2011; Srivastava et al., 2011). Several research groups have performed bioinformatic analyses, including gene ontology annotation of molecular function, biological processes and cellular components, and Kyoto Encyclopedia 
of Genes and Genomes (KEGG) pathways and predicted target genes, on mRNA or miRNA displaying altered expression. Despite these, research on the relationship between miRNA and the development of pancreatic adenocarcinoma using high throughput methodologies is extremely rare. This study provides important information to facilitate the elucidation of the physiological and pathological processes of pancreatic adenocarcinoma.

\section{MATERIAL AND METHODS}

\section{mRNA and miRNA microarray data}

GSE32676 and GSE32678, each containing 32 mRNA or miRNA expression chips from 25 early-stage pancreatic ductal adenocarcinoma (PDAC) samples and 7 non-malignant pancreatic samples, and uploaded by the same contributors, were downloaded from the gene expression omnibus (GEO) database.

\section{Preprocessing of microarray data}

mRNA and miRNA microarray data must be preprocessed prior to the identification of differentially expressed genes. Background correction and normalization was performed using the Affy package in R (R Core Team, 2013). Upon the detection of a gene by multiple probes, the mean expression value of those probes was utilized.

\section{Identification of differentially expressed genes and miRNA}

The Limma package in the R programming platform was used to identify the differentially expressed genes (DEGs) and miRNA. The Student $t$-test and the Bonferroni's correction method was used with an adjusted $\mathrm{P}$ value $<0.05$ and a $|\operatorname{logFC}|$ value $>1$.

\section{Functional annotation of DEGs}

Functional annotation of the DEGs was performed using the Database for Annotation, Visualization and Integrated Discovery (http://david.abcc.ncifcrf.gov/, DAVID). Gene ontology (GO) terms and KEGG pathways with $\mathrm{P}$ values $<0.05$ and at least 5 genes were selected.

\section{Screening of miRNA-mRNA relationships}

The miRNA-mRNA relationships were screened using the TarMir (http://www.tarmir. rgcb.res.in/) database. TarMir integrates a majority of the common miRNA databases, such as TargetScan, miRanda, and Point-in-Time Architecture (PITA), to identify the miRNA-mRNA relationships in a customizable and comprehensive manner. In this method, the miRNAmRNA relationships identified in DIANA, miRanda, PITA, and TargetScan were simultaneously retrieved by TarMir, the overlapped genes between DEGs and the target genes of differentially expressed miRNA were filtered, and the miRNA-mRNA regulatory network was constructed using those miRNA-mRNA relationships. The network was visualized using the Cytoscape platform software (Shannon et al., 2003). 


\section{RESULTS}

\section{Preprocessing of microarray data and identification of DEGs}

Normalized gene and miRNA expression profiles were obtained after background correction and the normalization of microarray data. The box plots of gene expression data before and after preprocessing are shown in Figure 1. Six hundred and twenty DEGs, including 441 up-regulated and 179 down-regulated genes, were identified by the Student $t$-test and Bonferroni's correction. Forty-eight differentially expressed miRNA (27 up-regulated and 21 downregulated miRNA) were also selected.
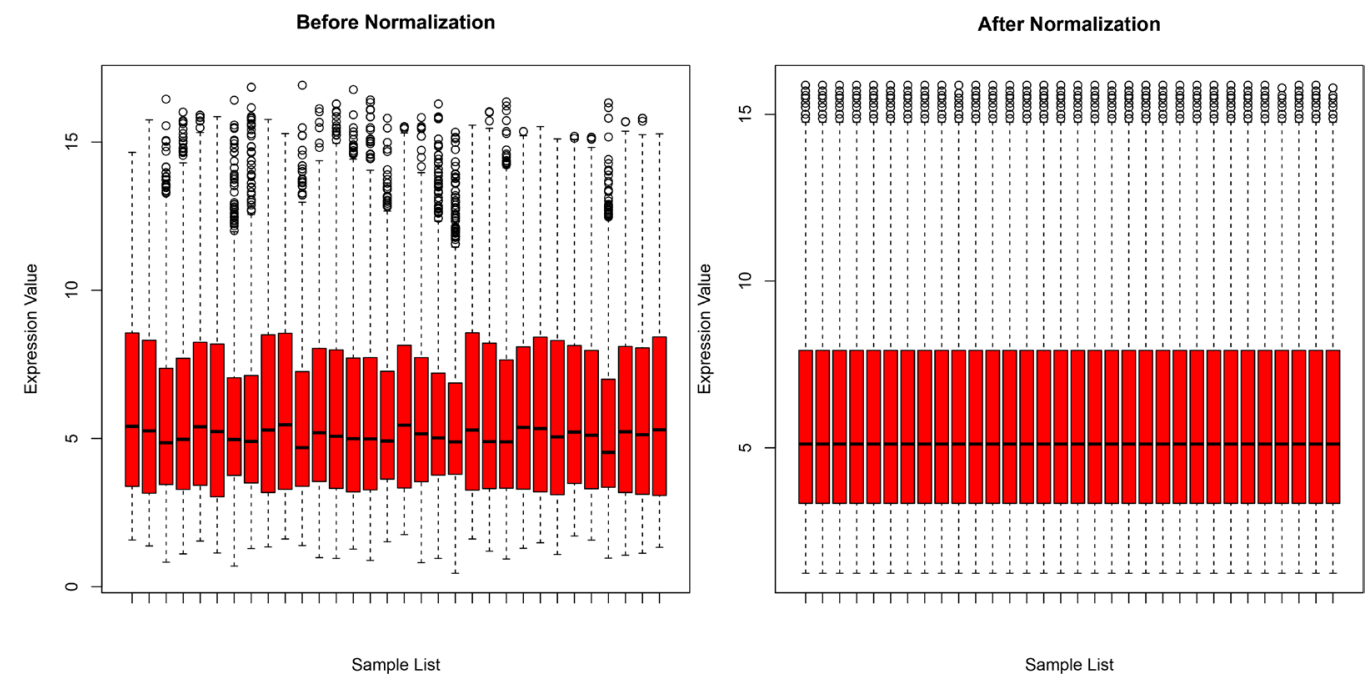

Figure 1. Expression value of all genes in every sample before and after normalization.

\section{Functional annotation of DEGs}

A total of $111 \mathrm{GO}$ terms, such as wound healing, cell adhesion, and biological adhesion, were enriched in the DEGs. In addition, 11 KEGG pathways, containing cell adhesion molecules, small cell lung cancer, and the p53 signaling pathway, which are well-researched aspects of cancers, were found to be significant (Table 1).

\section{miRNA-mRNA relationship}

Six thousand and nine hundred and thirty-one miRNA-mRNA regulatory relationships were retrieved from the TarMir database. Among these, 220 relationships were defined by DEGs and differentially expressed miRNA (Table 2). These were used to construct an miRNA-mRNA regulatory network (Figure 2) in PDAC. Corresponding degrees of genes/ miRNAs in the miRNA-mRNA regulatory network are shown in Table 3. 
Table 1. KEGG pathways of differentially expressed genes (DEGs).

\begin{tabular}{|c|c|c|c|c|}
\hline Category & Pathway name & No. of genes & $P$ value & Genes \\
\hline KEGG_PATHWAY & $\begin{array}{l}\text { O-glycan } \\
\text { biosynthesis }\end{array}$ & 7 & 4.02E-04 & $\begin{array}{l}\text { GALNT3, GCNT3, } \\
\text { GALNT7, GALNT5, } \\
\text { GALNT12, C1GALT1, } \\
\text { ST6GALNAC1 }\end{array}$ \\
\hline KEGG_PATHWAY & $\begin{array}{l}\text { Cell adhesion } \\
\text { molecules }\end{array}$ & 11 & 0.012884 & $\begin{array}{l}\text { F11R, CLDN7, SDC1, } \\
\text { PTPRF, ITGA6, } \\
C L D N 1, C D H 1, \\
C D H 3, \text { SDC4, SELE, } \\
C L D N 23\end{array}$ \\
\hline KEGG_PATHWAY & $\begin{array}{l}\text { Arrhythmogenic right } \\
\text { ventricular } \\
\text { cardiomyopathy } \\
\text { (ARVC) }\end{array}$ & 8 & 0.013361 & $\begin{array}{l}\text { JUP, DSG2, ITGA6, } \\
\text { ITGB6, ITGA2, } \\
\text { CACNB3, ITGA3, } \\
\text { TCF7L2 }\end{array}$ \\
\hline KEGG_PATHWAY & Tight junction & 11 & 0.014215 & $\begin{array}{l}\text { F11R, EPB41L3, } \\
C L D N 7, C T T N, C G N, \\
C L D N 1, \text { PRKCI, } \\
\text { MYH14, TJP3, } \\
C L D N 23, L L G L 2\end{array}$ \\
\hline KEGG_PATHWAY & Small cell lung cancer & 8 & 0.022157 & $\begin{array}{l}\text { LAMB3, CCND1, } \\
\text { ITGA6, PIAS3, } \\
\text { ITGA2, LAMC2, } \\
\text { ITGA3, MYC }\end{array}$ \\
\hline KEGG_PATHWAY & $\begin{array}{l}\text { ECM-receptor } \\
\text { interaction }\end{array}$ & 8 & 0.022157 & $\begin{array}{l}\text { LAMB3, SDC1, } \\
\text { ITGA6, ITGB6, } \\
\text { ITGA2, LAMC2, } \\
\text { ITGA3, SDC4 }\end{array}$ \\
\hline KEGG_PATHWAY & p53 signaling pathway & 7 & 0.025923 & $\begin{array}{l}\text { TP53I3, CCND1, } \\
\text { SERPINB5, RRM2, } \\
\text { SFN, PERP, } \\
\text { GADD } 45 B\end{array}$ \\
\hline KEGG_PATHWAY & Pathways in cancer & 21 & 0.026051 & $\begin{array}{l}\text { WNT5A, BMP4, IL6, } \\
\text { EGLN3, ITGA2, } \\
\text { EGLN3, ITGA2, } \\
\text { CDH1, ITGA3, } \\
\text { ZBTB16, MECOM, } \\
\text { TCF7L2, JUP, CBLC, } \\
\text { LAMB3, CCND1, } \\
\text { ITGA6, PIAS3, } \\
\text { LAMC2, MYC, } \\
\text { GSTP1 }\end{array}$ \\
\hline KEGG_PATHWAY & $\begin{array}{l}\text { Jak-STAT signaling } \\
\text { pathway }\end{array}$ & 11 & 0.03508 & $\begin{array}{l}\text { CSF3, CBLC, } \\
\text { CCND1, IL6, SOCS2, } \\
\text { PIAS3, SOCS3, PIM1, } \\
\text { LIFR, MYC, GHR }\end{array}$ \\
\hline KEGG_PATHWAY & $\begin{array}{l}\text { Acute myeloid } \\
\text { leukemia }\end{array}$ & 6 & 0.044307 & $\begin{array}{l}\text { JUP, CCND1, PIM1, } \\
\text { ZBTB16, MYC, } \\
\text { TCF7L2 }\end{array}$ \\
\hline KEGG_PATHWAY & $\begin{array}{l}\text { Aldosterone-regulated } \\
\text { sodium reabsorption }\end{array}$ & 5 & 0.047589 & $\begin{array}{l}\text { ATP1B1, SGK1, SFN, } \\
\text { SCNN1A, IRS1 }\end{array}$ \\
\hline
\end{tabular}

\section{DISCUSSION}

The pathogenesis of pancreatic adenocarcinoma remains poorly understood, which allows for the development of novel preventive and therapeutic interventions. In this study, the miRNA and mRNA expression profiles of patients with early-stage PDAC, as well as those of non-malignant pancreatic samples, were downloaded from gene databases. The miRNA (GSE32678) and mRNA (GSE32676) array deposited in the GEO database was analyzed, resulting in the identification of 620 DEGs from the original dataset, including 441 up-regulated and 179 down-regulated genes. 
Table 2. miRNA-mRNA regulatory relationship comprised of differentially expressed genes and differentially expressed microRNA (miRNA).

\begin{tabular}{|c|c|c|c|c|c|c|c|}
\hline miRNA & mRNA & miRNA & mRNA & miRNA & mRNA & miRNA & mRNA \\
\hline$h s a-m i R-135 b$ & ADAMTS9 & $h s a-m i R-429$ & $T F A P 2 A$ & hsa-miR-182 & $C T T N$ & $h s a-m i R-34 a$ & JAKMIPI \\
\hline hsa-miR-135b & CALML4 & hsa-miR-429 & TNFSF8 & hsa-miR-182 & $E F N B 2$ & $h s a-m i R-34 a$ & MARCKSLI \\
\hline hsa-miR-135b & $C D R 2 L$ & $h s a-m i R-425$ & $C R E M$ & hsa-miR-182 & ELMOI & $h s a-m i R-34 a$ & MYRIP \\
\hline$h s a-m i R-135 b$ & $E F N B 2$ & hsa-miR-425 & $E F N B 2$ & hsa-miR-182 & $F G R$ & $h s a-m i R-34 a$ & NHS \\
\hline hsa-miR-135b & $E M P 1$ & $h s a-m i R-425$ & MYRIP & hsa-miR-182 & $F N B P 1 L$ & $h s a-m i R-34 a$ & $N R 4 A 2$ \\
\hline hsa-miR-135b & GHR & hsa-miR-425 & SH3RF1 & hsa-miR-182 & KIAA1217 & $h s a-m i R-34 a$ & NRN1 \\
\hline hsa-miR-135b & PHF 17 & $h s a-m i R-222$ & CEP55 & hsa-miR-182 & MYRIP & $h s a-m i R-34 a$ & SOX4 \\
\hline hsa-miR-135b & $R A B 39 B$ & hsa-miR-222 & EFNB2 & hsa-miR-182 & NHS & $h s a-m i R-28-5 p$ & ANK2 \\
\hline hsa-miR-135b & TRPM4 & hsa-miR-222 & NAP1L2 & hsa-miR-182 & NRN1 & hsa-miR-28-5p & $B Z R A P I$ \\
\hline hsa-miR-200a & DCUN1D3 & hsa-miR-222 & NAPIL5 & hsa-miR-182 & TOX3 & hsa-miR-28-5p & $D C L K 1$ \\
\hline hsa-miR-200a & FAM $46 C$ & hsa-miR-222 & $P A K 1$ & $h s a-m i R-874$ & ARHGAP12 & $h s a-m i R-28-5 p$ & EFNA5 \\
\hline hsa-miR-200a & $F N B P I L$ & $h s a-m i R-489$ & EFNA5 & $h s a-m i R-874$ & DCUN1D3 & $h s a-m i R-28-5 p$ & HOXB3 \\
\hline hsa-miR-200a & HOXB5 & hsa-miR-489 & NFIL3 & $h s a-m i R-874$ & NHS & $h s a-m i R-28-5 p$ & $N R 4 A 3$ \\
\hline hsa-miR-200a & ITGA6 & hsa-miR-489 & $N R 4 A 3$ & hsa-miR-96 & $A N L N$ & hsa-miR-28-5p & PLEKHA7 \\
\hline hsa-miR-200a & MYRIP & hsa-miR-203 & $A C S L 1$ & hsa-miR-96 & CAMK2N1 & $h s a-m i R-28-5 p$ & SIPRI \\
\hline hsa-miR-200a & $R A B 27 B$ & $h s a-m i R-203$ & DCUN1D3 & hsa-miR-96 & $F O X Q 1$ & $h s a-m i R-28-5 p$ & SLC4A11 \\
\hline hsa-miR-200a & RHPN2 & hsa-miR-203 & DPY19L2 & hsa-miR-96 & GRHL2 & hsa-miR-488 & $A D A M 9$ \\
\hline hsa-miR-200a & $T F A P 2 A$ & $h s a-m i R-203$ & $F K B P I B$ & $h s a-m i R-96$ & IRSI & $h s a-m i R-488$ & $A D R B 2$ \\
\hline hsa-miR-200a & WNT5A & hsa-miR-203 & IRSI & hsa-miR-96 & MYRIP & $h s a-m i R-488$ & $K C N S 3$ \\
\hline$h s a-m i R-200 b$ & ADAMTS3 & hsa-miR-203 & ITGA2 & $h s a-m i R-96$ & $N R 4 A 3$ & $h s a-m i R-488$ & KIAA1217 \\
\hline$h s a-m i R-200 b$ & $A N L N$ & hsa-miR-203 & NFIL3 & hsa-miR-96 & PAKl & hsa-miR-488 & MXRA5 \\
\hline hsa-miR-200b & $C D R 2 L$ & $h s a-m i R-203$ & $N R 4 A 3$ & hsa-miR-96 & PROK2 & hsa-miR-488 & $P P A P 2 B$ \\
\hline$h s a-m i R-200 b$ & EFNA1 & hsa-miR-203 & $P P A P 2 B$ & hsa-miR-96 & RHPN2 & $h s a-m i R-488$ & $\mathrm{SH} 2 \mathrm{~B} 3$ \\
\hline hsa-miR-200b & $E F N B 2$ & $h s a-m i R-203$ & PREXI & $h s a-m i R-135 a$ & ADAMTS9 & $h s a-m i R-488$ & STK39 \\
\hline hsa-miR-200b & LMO7 & hsa-miR-203 & SOCS3 & $h s a-m i R-135 a$ & CALML4 & $h s a-m i R-526 a$ & ANK2 \\
\hline$h s a-m i R-200 b$ & $L R R C 8 A$ & $h s a-m i R-203$ & TOX3 & hsa-miR-135a & $C D R 2 L$ & hsa-miR-526a & $E F N B 2$ \\
\hline$h s a-m i R-200 b$ & MBOAT2 & hsa-miR-608 & $E B F 1$ & hsa-miR-135a & EFNB2 & hsa-miR-526a & $E G L N 3$ \\
\hline hsa-miR-200b & NAP1L2 & hsa-miR-608 & NRN1 & hsa-miR-135a & $E M P 1$ & hsa-miR-526a & HOXB3 \\
\hline hsa-miR-200b & NAP1L5 & hsa-miR-608 & PIP4K2C & hsa-miR-135a & $G H R$ & hsa-miR-141 & DCUN1D3 \\
\hline hsa-miR-200b & PHF 17 & hsa-miR-608 & $R A B 39 B$ & hsa-miR-135a & PHF 17 & $h s a-m i R-141$ & FAM46C \\
\hline$h s a-m i R-200 b$ & $P P A P 2 B$ & hsa-miR-183 & ENCl & hsa-miR-135a & $R A B 39 B$ & $h s a-m i R-141$ & $F N B P 1 L$ \\
\hline$h s a-m i R-200 b$ & PROK2 & hsa-miR-183 & $I R S I$ & hsa-miR-135a & TRPM4 & $h s a-m i R-141$ & HOXB5 \\
\hline hsa-miR-200b & PVRL4 & hsa-miR-183 & KIAA0101 & $h s a-m i R-34 c-5 p$ & ACSL1 & $h s a-m i R-141$ & ITGA6 \\
\hline$h s a-m i R-200 b$ & TFAP $2 A$ & hsa-miR-183 & PIMI & $h s a-m i R-34 c-5 p$ & ACSL4 & hsa-miR-141 & MYRIP \\
\hline hsa-miR-200b & TNFSF8 & hsa-miR-183 & TCF $7 L 2$ & $h s a-m i R-34 c-5 p$ & ANK2 & hsa-miR-141 & $R A B 27 B$ \\
\hline hsa-miR-21 & GRAMD3 & hsa-miR-200c & ADAMTS3 & $h s a-m i R-34 c-5 p$ & CBFA2T3 & hsa-miR-141 & RHPN2 \\
\hline hsa-miR-21 & LIFR & $h s a-m i R-200 c$ & $A N L N$ & $h s a-m i R-34 c-5 p$ & $D K K 1$ & hsa-miR-141 & SOXO \\
\hline hsa-miR-21 & MYO6 & $h s a-m i R-200 c$ & $C D R 2 L$ & $h s a-m i R-34 c-5 p$ & $F K B P I B$ & hsa-miR-141 & TFAP $2 A$ \\
\hline hsa-miR-21 & PHF17 & $h s a-m i R-200 c$ & EFNA1 & $h s a-m i R-34 c-5 p$ & $F O X Q 1$ & hsa-miR-141 & WNT5A \\
\hline hsa-miR-21 & PLEKHAI & $h s a-m i R-200 c$ & EFNB2 & $h s a-m i R-34 c-5 p$ & GALNT7 & $h s a-m i R-92 b$ & ADAMIO \\
\hline hsa-miR-21 & SASHI & $h s a-m i R-200 c$ & LMO7 & $h s a-m i R-34 c-5 p$ & $J A K M I P 1$ & hsa-miR-92b & CNNM4 \\
\hline hsa-miR-429 & ADAMTS3 & $h s a-m i R-200 c$ & LRRC8A & $h s a-m i R-34 c-5 p$ & MARCKSL1 & $h s a-m i R-92 b$ & DNAJB9 \\
\hline hsa-miR-429 & $A N L N$ & $h s a-m i R-200 c$ & $M B O A T 2$ & $h s a-m i R-34 c-5 p$ & MYRIP & $h s a-m i R-92 b$ & $E D N R B$ \\
\hline hsa-miR-429 & $C D R 2 L$ & $h s a-m i R-200 c$ & NAP1L2 & $h s a-m i R-34 c-5 p$ & NHS & $h s a-m i R-92 b$ & $G A L N T 7$ \\
\hline$h s a-m i R-429$ & EFNA1 & $h s a-m i R-200 c$ & NAPIL5 & $h s a-m i R-34 c-5 p$ & NR4A2 & $h s a-m i R-92 b$ & GHR \\
\hline$h s a-m i R-429$ & EFNB2 & $h s a-m i R-200 c$ & PHF17 & $h s a-m i R-34 c-5 p$ & NRN1 & $h s a-m i R-92 b$ & GRAMD3 \\
\hline hsa-miR-429 & LMO7 & hsa-miR-200c & $P P A P 2 B$ & $h s a-m i R-34 c-5 p$ & SOX4 & hsa-miR-92b & $N R 4 A 3$ \\
\hline hsa-miR-429 & LRRC8A & $h s a-m i R-200 c$ & PROK2 & $h s a-m i R-34 a$ & ACSL1 & $h s a-m i R-92 b$ & PLEKHAI \\
\hline hsa-miR-429 & MBOAT2 & $h s a-m i R-200 c$ & PVRL4 & hsa-miR-34a & ACSL4 & $h s a-m i R-92 b$ & $P P A P 2 B$ \\
\hline hsa-miR-429 & NAP1L2 & $h s a-m i R-200 c$ & TFAP $2 A$ & $h s a-m i R-34 a$ & ANK2 & $h s a-m i R-92 b$ & SIPRI \\
\hline hsa-miR-429 & NAP1L5 & $h s a-m i R-200 c$ & TNFSF 8 & $h s a-m i R-34 a$ & CBFA2T3 & $h s a-m i R-92 b$ & SOX4 \\
\hline hsa-miR-429 & PHF17 & hsa-miR-671-5p & PHF17 & hsa-miR-34a & $D K K 1$ & $h s a-m i R-92 b$ & STK39 \\
\hline hsa-miR-429 & $P P A P 2 B$ & hsa-miR-182 & ADAMIO & hsa-miR-34a & $F K B P 1 B$ & hsa-miR-552 & DCUN1D3 \\
\hline hsa-miR-429 & PROK2 & hsa-miR-182 & $A N K 2$ & $h s a-m i R-34 a$ & $F O X Q 1$ & $h s a-m i R-552$ & ENCl \\
\hline hsa-miR-429 & PVRL4 & hsa-miR-182 & CBFA2T3 & hsa-miR-34a & GALNT7 & hsa-miR-552 & $S G K 1$ \\
\hline
\end{tabular}

GO analysis revealed many significantly enriched biological processes, including wound response and healing, cell adhesion, biological adhesion, inflammatory response, and response to corticosteroid stimulus. The KEGG pathway-enrichment analysis revealed a sig- 
nificant overexpression of the o-glycan biosynthesis pathway, cell adhesion molecules, arrhythmogenic right ventricular cardiomyopathy, and tight junction, in the early-stage PDAC samples. Pancreatic ductal adenocarcinoma is characterized by early loco-regional spread and distant metastasis. The development of metastasis is determined by the gradual increase in essential changes in cancerous cells, as well as their communications with different stromal elements in the tumor microenvironment. Cell adhesion and biological adhesion play significant roles in cancer metastasis. Moreover, the inflammatory response and the migration of the myeloid (macrophages, dendritic cells, neutrophils, myeloid-derived suppressor cells) and lymphoid (regulatory T, B and NK cells) immune regulatory cells to the tumor site have been reported to support tumor growth, spread of tumor, and tumor metastasis (Keskinov and Shurin, 2014).

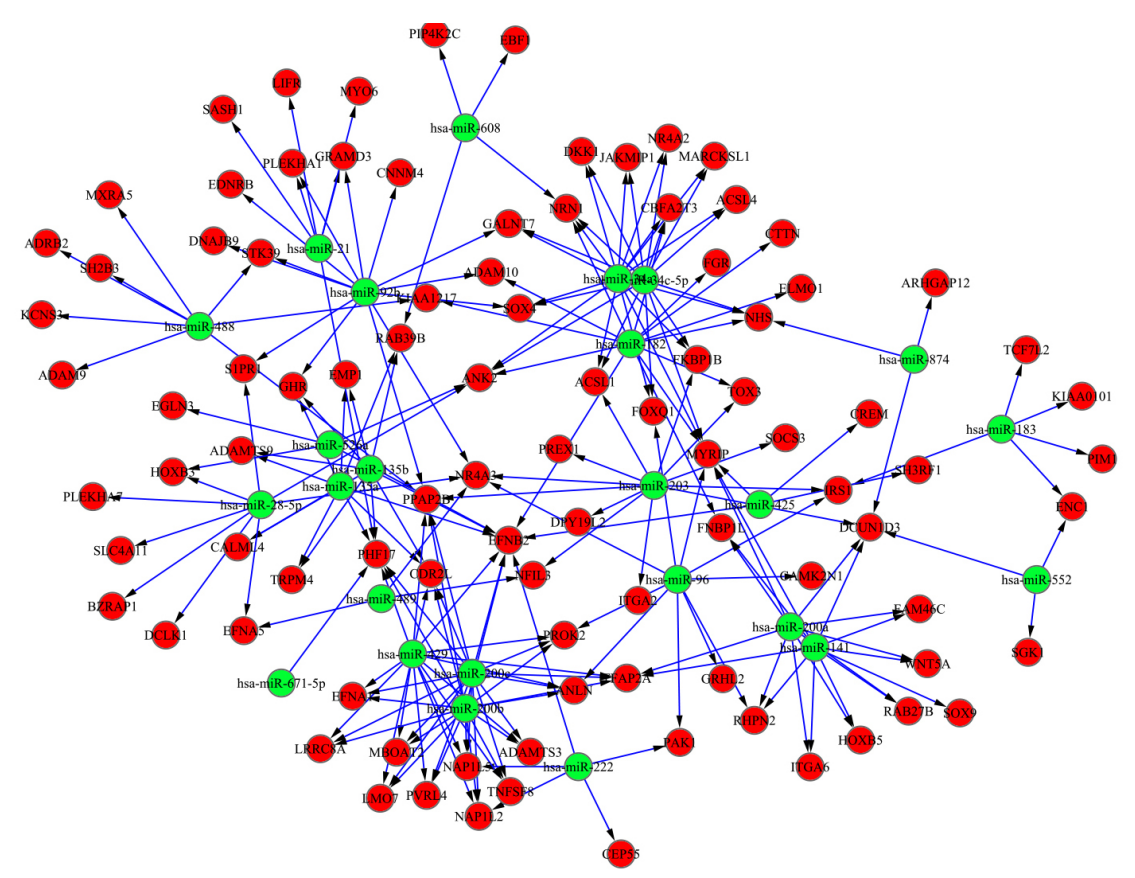

Figure 2. miRNA-mRNA regulation network. Red nodes represent genes and bright green nodes represent miRNAs.

The target genes regulated by the differentially expressed miRNA and mRNA were then identified and further analyzed, in order to define their relationship with miRNA and mRNA; subsequently, an miRNA-mRNA regulatory network was constructed.

A thorough analysis of the regulatory network revealed an extremely high node degree of hsa-miR-200c, hsa-miR-429, and hsa-miR-200b (miRNA), and EFNB2, MYRIP, and PHF17 (mRNA), which indicated that these miRNA and mRNA may play a key role in the development of pancreatic cancer. The miR-200 family (miR-200a, miR-200b, miR-200c, $m i R-141$, and $m i R-429)$ is a cluster of miRNA that are highly correlated with epithelial-mesenchymal transition, with $m i R-200 b$ being identified as a critical regulator of tumor invasion, 


\section{Table 3. Genes/microRNA (miRNA) in the miRNA-mRNA regulatory network and their corresponding degree.}

\begin{tabular}{|c|c|c|c|}
\hline Node & Degree & Node & Degree \\
\hline hsa-miR-671-5p & 1 & $H O X B 3$ & 2 \\
\hline LIFR & 1 & SIPRI & 2 \\
\hline MYO6 & 1 & STK39 & 2 \\
\hline SASHI & 1 & hsa-miR-489 & 3 \\
\hline CREM & 1 & hsa-miR-874 & 3 \\
\hline$S H 3 R F 1$ & 1 & hsa-miR-552 & 3 \\
\hline CEP55 & 1 & $G H R$ & 3 \\
\hline DPY19L2 & 1 & $R A B 39 B$ & 3 \\
\hline$I T G A 2$ & 1 & FNBPIL & 3 \\
\hline PREXI & 1 & RHPN2 & 3 \\
\hline SOCS3 & 1 & ADAMTS3 & 3 \\
\hline$E B F 1$ & 1 & EFNAI & 3 \\
\hline$P I P 4 K 2 C$ & 1 & LMO7 & 3 \\
\hline KIAA0101 & 1 & $L R R C 8 A$ & 3 \\
\hline PIMI & 1 & MBOAT2 & 3 \\
\hline$T C F 7 L 2$ & 1 & PVRL4 & 3 \\
\hline$C T T N$ & 1 & TNFSF8 & 3 \\
\hline ELMOI & 1 & $A C S L 1$ & 3 \\
\hline$F G R$ & 1 & $F K B P 1 B$ & 3 \\
\hline ARHGAP12 & 1 & IRSI & 3 \\
\hline CAMK2N1 & 1 & $C B F A 2 T 3$ & 3 \\
\hline GRHL2 & 1 & $F O X Q 1$ & 3 \\
\hline$B Z R A P 1$ & 1 & GALNT7 & 3 \\
\hline$D C L K 1$ & 1 & SOX4 & 3 \\
\hline PLEKHA7 & 1 & hsa-miR-425 & 4 \\
\hline SLC4A11 & 1 & hsa-miR-608 & 4 \\
\hline$A D A M 9$ & 1 & $h s a-m i R-526 a$ & 4 \\
\hline$A D R B 2$ & 1 & $A N L N$ & 4 \\
\hline$K C N S 3$ & 1 & NAPIL2 & 4 \\
\hline$M X R A 5$ & 1 & NAPIL5 & 4 \\
\hline $\mathrm{SH} 2 \mathrm{~B} 3$ & 1 & PROK2 & 4 \\
\hline$E G L N 3$ & 1 & NRN1 & 4 \\
\hline SOXY & 1 & NHS & 4 \\
\hline CNNM4 & 1 & hsa-miR-222 & 5 \\
\hline DNAJB 9 & 1 & hsa-miR-183 & 5 \\
\hline$E D N R B$ & 1 & $C D R 2 L$ & 5 \\
\hline$S G K 1$ & 1 & DCUNID3 & 5 \\
\hline ADAMTS9 & 2 & TFAP $2 A$ & 5 \\
\hline CALML4 & 2 & $N R 4 A 3$ & 5 \\
\hline EMP1 & 2 & ANK2 & 5 \\
\hline TRPM4 & 2 & hsa-miR-21 & 6 \\
\hline FAM46C & 2 & $P P A P 2 B$ & 6 \\
\hline HOXB5 & 2 & PHF17 & 7 \\
\hline ITGA6 & 2 & MYRIP & 7 \\
\hline$R A B 27 B$ & 2 & hsa-miR-488 & 8 \\
\hline WNT5A & 2 & $h s a-m i R-135 b$ & 9 \\
\hline GRAMD3 & 2 & $h s a-m i R-135 a$ & 9 \\
\hline PLEKHAI & 2 & $h s a-m i R-28-5 p$ & 9 \\
\hline$P A K 1$ & 2 & EFNB2 & 9 \\
\hline EFNA5 & 2 & $h s a-m i R-200 a$ & 10 \\
\hline NFIL3 & 2 & hsa-miR-96 & 10 \\
\hline TOX3 & 2 & hsa-miR-141 & 11 \\
\hline ENCl & 2 & hsa-miR-203 & 12 \\
\hline ADAMIO & 2 & hsa-miR-182 & 13 \\
\hline KIAA1217 & 2 & $h s a-m i R-92 b$ & 13 \\
\hline ACSL4 & 2 & $h s a-m i R-34 c-5 p$ & 15 \\
\hline$D K K 1$ & 2 & $h s a-m i R-34 a$ & 15 \\
\hline$J A K M I P 1$ & 2 & $h s a-m i R-200 b$ & 16 \\
\hline MARCKSL1 & 2 & $h s a-m i R-429$ & 16 \\
\hline NR4A2 & 2 & $h s a-m i R-200 c$ & 16 \\
\hline
\end{tabular}

The degree of genes is the number of miRNA that directly interact with it, while the degree of miRNA is denoted by the number of genes that directly interact with it. 
metastasis, and chemosensitivity (Feng et al., 2012). The miR-200 family is associated with the acquisition of epithelial-to-mesenchymal transition and gemcitabine sensitivity in pancreatic adenocarcinoma (Li et al., 2009; Ali et al., 2010; Bao et al., 2011). Previous studies have reported the close association of EFNB2 with the development of gastric cancer, neuroblastomas, esophageal squamous cell carcinoma, and other tumors (Tang et al., 1999, 2000; Kataoka et al., 2002; Tachibana et al., 2007). However, the relationship between specific genes and pancreatic adenocarcinoma has been rarely reported. We believe that these genes could be potential targets for the diagnosis and treatment of pancreatic adenocarcinoma. In summary, this study provides important information to facilitate the elucidation of the physiological and pathological processes governing pancreatic adenocarcinoma. However, the roles played by these genes in pancreatic adenocarcinoma must be further validated in vitro and in vivo, using the latest molecular biology techniques.

\section{Conflicts of interest}

The authors declare no conflict of interest.

\section{ACKNOWLEDGMENTS}

Research supported by the Health Bureau Science and Technology Foundation of Tianjin (\#2012KZ063) and the National Natural Science Foundation of China (Grants \#81302082, \#81272685, \#31301151, and \#81172355).

\section{REFERENCES}

Ali S, Ahmad A, Banerjee S, Padhye S, et al. (2010). Gemcitabine sensitivity can be induced in pancreatic cancer cells through modulation of miR-200 and miR-21 expression by curcumin or its analogue CDF. Cancer Res. 70: 36063617.

Antonini D, Russo MT, De Rosa L, Gorrese M, et al. (2010). Transcriptional repression of miR-34 family contributes to p63-mediated cell cycle progression in epidermal cells. J. Invest. Dermatol. 130: 1249-1257.

Bao B, Ali S, Kong D, Sarkar SH, et al. (2011). Anti-tumor activity of a novel compound-CDF is mediated by regulating miR-21, miR-200, and PTEN in pancreatic cancer. PLoS One 6: e17850.

Feng B, Wang R and Chen LB (2012). Review of miR-200b and cancer chemosensitivity. Biomed. Pharmacother. 66: 397-402.

Giovannetti E, Funel N, Peters GJ, Del Chiaro M, et al. (2010). MicroRNA-21 in pancreatic cancer: correlation with clinical outcome and pharmacologic aspects underlying its role in the modulation of gemcitabine activity. Cancer Res. 70: 4528-4538.

Kataoka H, Tanaka M, Kanamori M, Yoshii S, et al. (2002). Expression profile of EFNB1, EFNB2, two ligands of EPHB2 in human gastric cancer. J. Cancer Res. Clin. Oncol. 128: 343-348.

Keskinov AA and Shurin MR (2014). Myeloid regulatory cells in tumor spreading and metastasis. Immunobiology 220: 236-242.

Lee RC, Feinbaum RL and Ambros V (1993). The C. elegans heterochronic gene lin-4 encodes small RNAs with antisense complementarity to lin-14. Cell 75: 843-854.

Li Y, VandenBoom TG II, Kong D, Wang Z, et al. (2009). Up-regulation of miR-200 and let-7 by natural agents leads to the reversal of epithelial-to-mesenchymal transition in gemcitabine-resistant pancreatic cancer cells. Cancer Res. 69: 6704-6712.

Lionetti M, Biasiolo M, Agnelli L, Todoerti K, et al. (2009). Identification of microRNA expression patterns and definition of a microRNA/mRNA regulatory network in distinct molecular groups of multiple myeloma. Blood 114: e20-e26.

Liu C, Kelnar K, Liu B, Chen X, et al. (2011). The microRNA miR-34a inhibits prostate cancer stem cells and metastasis by directly repressing CD44. Nat. Med. 17: 211-215. 
Long J, Luo G, Liu C, Cui X, et al. (2012). Development of a unique mouse model for pancreatic cancer lymphatic metastasis. Int. J. Oncol. 41: 1662-1668.

Matano E, Tagliaferri P, Libroia A, Damiano V, et al. (2000). Gemcitabine combined with continuous infusion 5 -fluorouracil in advanced and symptomatic pancreatic cancer: a clinical benefit-oriented phase II study. $B r . J$. Cancer 82: 1772-1775.

R Core Team (2013). R: A language and environment for statistical computing. R Foundation for Statistical Computing, Austria. Available at [http://www.R-project.org/].

Ryu JK, Hong SM, Karikari CA, Hruban RH, et al. (2010). Aberrant MicroRNA-155 expression is an early event in the multistep progression of pancreatic adenocarcinoma. Pancreatology 10: 66-73.

Shannon P, Markiel A, Ozier O, Baliga NS, et al. (2003). Cytoscape: a software environment for integrated models of biomolecular interaction networks. Genome Res. 13: 2498-2504.

Siegel R, Ma J, Zou Z and Jemal A (2014). Cancer statistics 2012. CA Cancer J. Clin. 64: 364.

Srivastava SK, Bhardwaj A, Singh S, Arora S, et al. (2011). MicroRNA-150 directly targets MUC4 and suppresses growth and malignant behavior of pancreatic cancer cells. Carcinogenesis 32: 1832-1839.

Tachibana M, Tonomoto Y, Hyakudomi R, Hyakudomi M, et al. (2007). Expression and prognostic significance of EFNB2 and EphB4 genes in patients with oesophageal squamous cell carcinoma. Dig. Liver Dis. 39: 725-732.

Tang XX, Evans AE, Zhao H, Cnaan A, et al. (1999). High-level expression of EPHB6, EFNB2, and EFNB3 is associated with low tumor stage and high TrkA expression in human neuroblastomas. Clin. Cancer Res. 5: 1491-1496.

Tang XX, Zhao H, Robinson ME, Cohen B, et al. (2000). Implications of EPHB6, EFNB2, and EFNB3 expressions in human neuroblastoma. Proc. Natl. Acad. Sci. 97: 10936-10941. 